\title{
Dietary patterns of takeaway (fast) food consumers in the North West of England: A Pilot study
}

\author{
H.G. Janssen, I.G. Davies, L. Richardson and L. Stevenson \\ School of Sport Studies, Leisure and Nutrition, Liverpool John Moores University, Barkhill Road, Liverpool, \\ $L 17$ 6BD, UK.
}

Takeaway (fast) foods have become increasingly popular and are thought to be associated with a number of non-communicable diseases $^{(1)}$ owing to their high fat, salt and sugar content ${ }^{(2)}$. In recent years, research into dietary intake has shifted from analyses of single foods or nutrients to a more complex assessment of the whole diet to produce dietary patterns. Dietary patterns may be more predictive of disease risk than individual foods or nutrients due to the synergistic effects of food combinations ${ }^{(3,4)}$. The aim of the present study was elucidate the dietary patterns of takeaway (fast) food consumers in the North West of England and the factors influencing their consumption. The objectives were to measure habitual diet and takeaway (fast) food intake, identify novel dietary patterns, and assess whether frequent takeaway (fast) food consumers had a higher intake of unhealthy foods in their habitual diet.

The Research Ethics Committee at Liverpool John Moores University granted ethical approval. A modified version of the EPIC-Norfolk food frequency questionnaire, to include takeaway (fast) food consumption and socio-demographics, was created, tested and optimised in a pilot study $(n=26)$. Data from the food frequency questionnaire was converted into portions per week and principal component analysis was performed to identify dietary patterns in the study sample.

Results revealed three distinct dietary patterns (characterised as 'Traditional, 'Cosmopolitan' and 'Convenience') (Fig. 1). The three-component solution explained $42.9 \%$ of the variance in dietary intake. A particularly interesting finding was the elements of the diet that tracked together in the 'convenience' dietary pattern including unhealthy habitual foods (refined grains, cakes, pastries etc.) and takeaway (fast) food (Chinese, Indian, kebabs etc.). In conclusion, the findings support the concept that takeaway (fast) food consumption is a marker of an unhealthy diet overall.

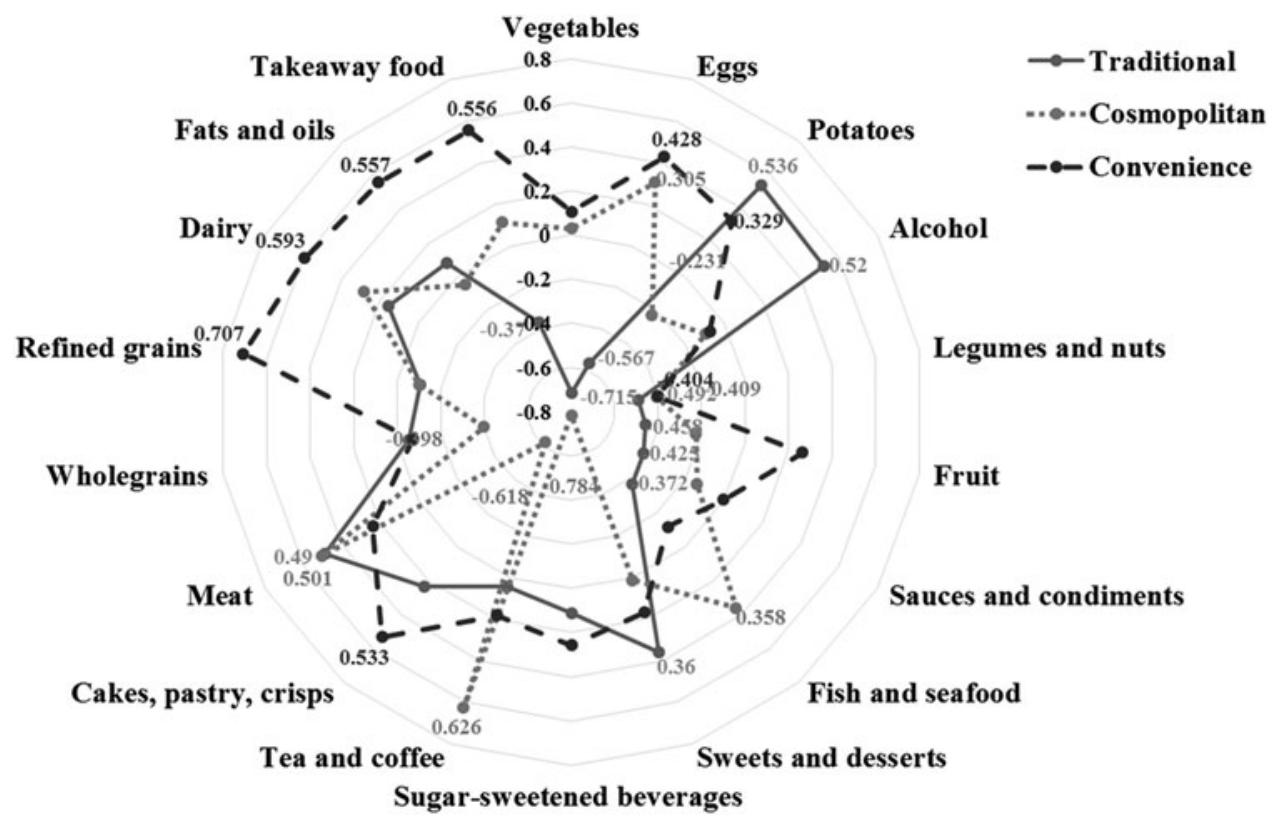

Fig. 1. Dietary patterns and factor loadings derived through principal component analysis.

1. Kant AK, Whitley MI \& Graubard BI. (2015) Int J Obes. 39, 820-827.

2. Jaworowska A, Blackham TM, Long R, et al. (2014) Nutr Food Sci. 44, 414-430.

3. Granic A, Davies K, Adamson A, et al. (2015) PLoS One. 10, e0139713.

4. Hu FB (2002) Curr Opin Lipidol. 13, 3-9. 